\title{
THG of ZnO nanorods for efficient third order interferometric FROG
}

\author{
Susanta Kumar Das ${ }^{1,4}$, Frank Güell ${ }^{2}$, Ciarán Gray ${ }^{3}$, Prasanta Kumar Das ${ }^{4}$, Ruediger Grunwald ${ }^{1}$, Enda \\ McGlynn $^{3}$, and Günter Steinmeyer ${ }^{1}$ \\ ${ }^{1}$ Max-Born-Institut für Nichtlineare Optik und Kurzzeitspektroskopie, Max-Born-Strasse 2a, D-12489 Berlin, Germany. \\ ${ }^{2}$ Departament d'Electrònica, Universitat de Barcelona, C/Martí i Franquès 1, E-08028 Barcelona, Catalunya, Spain. \\ ${ }^{3}$ School of Physical Sciences, National Centre for Plasma Science and Technology, Dublin City University, Glasnevin, Dublin 9, Ireland. \\ ${ }^{4}$ School of Applied Sciences, KIIT University, Bhubaneswar - 751024, Odisha, India \\ email: steinmey@mbi-berlin.de
}

\begin{abstract}
Efficient third harmonic generation was found in $\mathrm{ZnO}$ nanorod layers grown by phase transport and low temperature chemical bath deposition method. Interferometric frequencyresolved optical gating of few cycle fs pulses was demonstrated.

OCIS codes: (160.4236) Nanomaterials; (190.4400) Nonlinear optics, materials; (190.7110) Ultrafast nonlinear optics.
\end{abstract}

$\mathrm{ZnO}$ nanostructures are of increasing interest for optoelectronics, biomedicine, and other applications. Depending on the particular growth method, they appear in various nanomorphologies with specific physical and chemical properties [1]. High temperature vapour phase transport (VPT) yields material with excellent emission properties but is limited with respect to the substrate size. $\mathrm{ZnO}$ from chemical bath deposition (CBD) at lower temperatures can be obtained with better uniformity on larger areas yet low efficiency photoluminescence (PL). The relationship between structure and nonlinear properties for complex nanomaterials, in particular third harmonic generation (THG) [2,3], is still less understood. The enhancement of THG in structured nanolayers was demonstrated to enable the complete characterization of ultrashort laser pulses at low dispersion and without the necessity of phase matching because of a crystal thickness well below the coherence length [4].

Here we report on the efficient generation of THG with different types of $\mathrm{ZnO}$ nanorods grown by both VPT and $\mathrm{CBD}$ and third order interferometric frequency-resolved optical gating (iFROG) [5]. ZnO nanorods of comparable geometry and size were grown by CBD and VPT on fused silica and (11-20) sapphire substrates, respectively [6]. The samples were characterized after deposition by field emission scanning electron microscopes (SEM) (JEOL JSM-6400F, Hitachi H-4100FE) and X-ray diffraction (XRD; Bruker AXS D8 Advance Texture Diffractometer). The SEM micrographs in Figs. 1 (a),(b) show the structure of the densely packed nanorod layers.
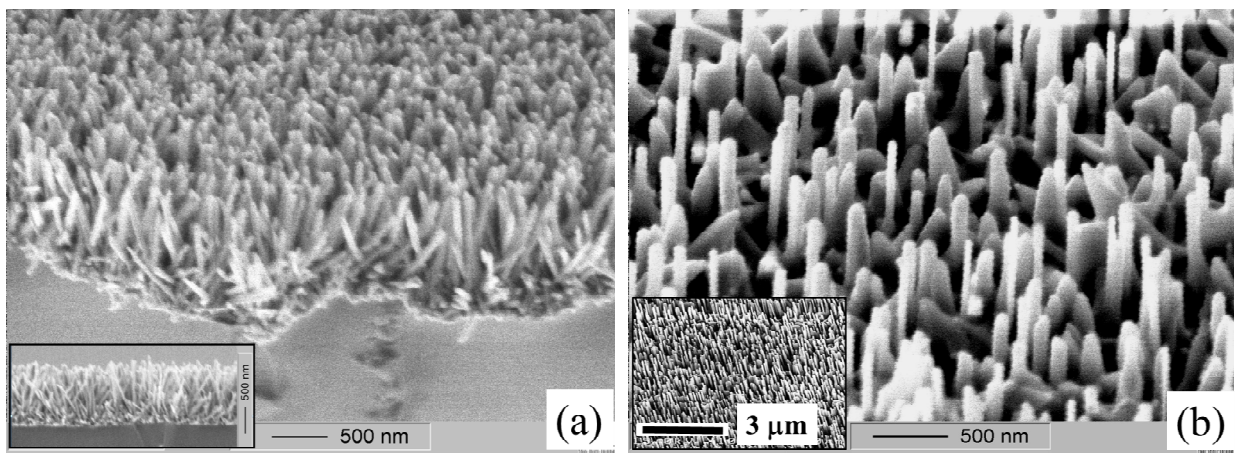

Fig. 1. (a) FESEM data ( $45^{\circ}$ tilted-view image) of the CBD grown $\mathrm{ZnO}$ nanorod sample (inset, crosssectional view); (b) FESEM data ( $45^{\circ}$ tilted-view image) of the VPT-grown $\mathrm{ZnO}$ nanorod sample grown on sapphire (inset, lower resolution image showing wider field of view).

For the frequency conversion experiments, a Ti:sapphire laser oscillator (VENTEON) was used (pulse duration 6-7 fs, center wavelength $810 \mathrm{~nm}$, repetition rate $80 \mathrm{MHz}$, pulse energy $>5 \mathrm{~nJ}$, spetral FWHM bandwidth $>300 \mathrm{~nm}$ ). The beam waist in the focus was $4 \mu \mathrm{m}$, yielding a peak intensity of $\sim 1.5 \times 10^{12} \mathrm{~W} / \mathrm{cm}^{2}$ of the un-attenuated beam. iFROG measurements were performed using a home-made Michelson interferometer (piezo: $35 \mathrm{~nm}$ steps). The THG signal was separated from residual pump radiation by a THG reflecting mirror and an interference filter. The light 
was subsequently analyzed by a fiber-coupled spectrometer (Shamrock, LOT/Andor) with high sensitivity electron multiplier charge coupled device (EMCCD, Newton, LOT/Andor, cooled down to $-75^{\circ} \mathrm{C}$ ). The interferometer scan and the spectrum capture were synchronized via LabView. For the iFROG data analysis after Fourier filtering, commercial software (Femtosoft Technologies, FROG 3.0) was applied. Independent reference measurements of the spectral phase were performed with a Few-cycle Spectral Phase Interferometry for Direct Electric-field Reconstruction (FC-SPIDER, APE).

Figure 2 shows the third-order iFROG raw data from the CBD grown $\mathrm{ZnO}$ nanorods as well as retrieved conventional FROG plots and temporal pulse structure. The results from VPT grown nanorods are very similar (apart from a long wavelength shoulder). The different order frequency components were selected by Fourier filtering. The first two orders (i.e., DC and first order) were used to retrieve the pulse shape by two independent methods for consistency check, in particular for calibrating the delay axis. Here we restrict ourselves to analyzing the DC part of the trace that we extract by Fourier filtering. The resulting traces are shown in Figs. 2(b) and 2(e) for the case of the CBD and VPT grown samples, respectively. This trace is equivalent to an ordinary frequency resolved-optical gating (FROG) trace and hence can be directly be processed by standard pulse retrieval software. The retrieved spectral signal and spectral phase are shown in Figs. 2(c),(f). The corresponding final pulse duration is estimated to be $\sim 11 \mathrm{fs}$ in both cases (due to the use of an unbalanced beam splitter).
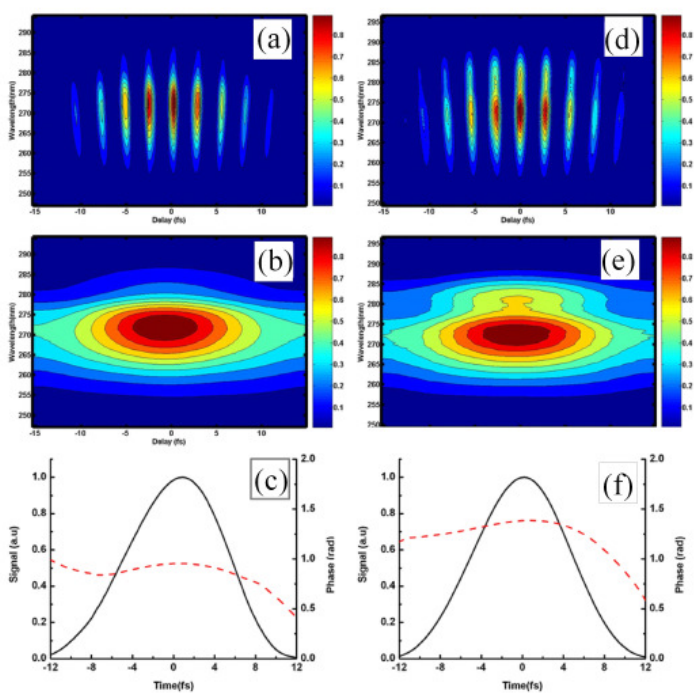

Fig. 2. (a) iFROG trace, (b) FROG trace, and (c) temporal pulse profile measured on $\mathrm{ZnO}$ nanorods grown by the CBD method; (d) iFROG trace, (e) FROG trace and (f) temporal pulse profile measured on $\mathrm{ZnO}$ nanorods grown by VPT method.

Taking into account the group delay dispersion (GDD) of the beam splitter, the pulse duration was corrected, and a value of $\tau \mathrm{o}=7 \mathrm{fs}$ was estimated, close to the value of $6.7 \mathrm{fs}$. Furthermore, highly dispersive media were inserted to add a defined chirp. For a GDD of $\beta_{2}=90 \mathrm{fs}^{2}$ (corresponding to stretching of the pulse duration to $40 \mathrm{fs}$ ), the accuracy of the retrieval of pulse durations was found to be in the $10 \%$ range.

To conclude, the results demonstrate the capability of CBD and VPT grown ZnO nanorods to serve as efficient, low-dispersion materials for nonlinear pulse characterization on sub-10-fs time scale. In contrast to their linearoptical properties, the THG efficiencies of both different types of samples are comparable. The structurally enhanced THG of $\mathrm{ZnO}$ nanorods may also stimulate applications like photodynamic therapy or multiphoton near field microscopy. The work was supported by LaserLab Europe (MBI 001954).

[1] A. B. Djurisic, Y. H. Leung, “Optical properties of ZnO nanostructures,” Small 2, 944-961 (2006).

[2] G. I. Petrov, V. Shcheslavskiy, V. V. Yakovlev, I. Ozerov, E. Chelnokov, W. Marine, "Efficient third-harmonic generation in a thin nanocrystalline film of ZnO,” Appl. Phys. Lett. 83, 3993-3995 (2003).

[3] K. Wang, J. Zhou, L.Y. Yuan, Y.T. Tao, J. Chen, P.X. Lu, Z.L. Wang, “Anisotropic third-order optical nonlinearity of a single ZnO micro/nanowire," Nano Lett. 12, 833-838 (2012).

[4] S.K. Das, C. Schwanke, A. Pfuch, W. Seeber, M. Bock, G. Steinmeyer, T. Elsaesser, R. Grunwald, "Highly efficient THG in TiO2 nanolayers for third-order pulse characterization," Opt. Express 19, 16985-16995 (2011).

[5] G. Stibenz, G. Steinmeyer, "Interferometric frequency-resolved optical gating," Opt. Express 13, 2617-2626 (2005).

[6] S. K. Das, F. Güell, C. Gray, P. Das, R. Grunwald, and E. Mc Glynn, ZnO nanorods for efficient third harmonic UV generation, Opt. Mat. Express (2014), submitted. 